\title{
Optimal Receiver Design for Diffusive Molecular Communication With Flow and Additive Noise
}

\author{
Adam Noel, Student Member, IEEE, Karen C. Cheung, and Robert Schober, Fellow, IEEE
}

\begin{abstract}
In this paper, we perform receiver design for a diffusive molecular communication environment. Our model includes flow in any direction, sources of information molecules in addition to the transmitter, and enzymes in the propagation environment to mitigate intersymbol interference. We characterize the mutual information between receiver observations to show how often independent observations can be made. We derive the maximum likelihood sequence detector to provide a lower bound on the bit error probability. We propose the family of weighted sum detectors for more practical implementation and derive their expected bit error probability. Under certain conditions, the performance of the optimal weighted sum detector is shown to be equivalent to a matched filter. Receiver simulation results show the tradeoff in detector complexity versus achievable bit error probability, and that a slow flow in any direction can improve the performance of a weighted sum detector.
\end{abstract}

Index Terms-Diffusion, matched filter, molecular communication, sequence detection, weighted sum detector.

\section{INTRODUCTION}

M OLECULAR communication is a promising design strategy for the transfer of information between devices that have functional components on the order of nanometers in size. The ability for such devices to communicate would significantly expand their cumulative potential, thereby enabling applications such as cooperative diagnostics and drug delivery in biomedicine, bottom-up component design in manufacturing, and sensitive environmental monitoring; see [1], [2]. Molecular communication uses physical molecules as information carriers, which are released by the transmitter and propagate to an intended receiver. Propagation methods include active schemes, where energy is consumed to direct the information molecules towards the receiver, and passive schemes, where the transmitter has no direct influence on the path taken by the released molecules. Passive schemes, such as free diffusion, are simpler to implement and more easily enable the formation of ad hoc networks between devices.

Diffusion-based communication is commonly found in cellular systems when small molecules need to quickly travel short distances; see [3, Ch. 4]. Due to its simplicity and its current implementation in nature, diffusion has also often been chosen by communication researchers for the design of synthetic nanonetworks. The diffusive channel has a number

Manuscript received July 31, 2013; revised June 18, 2014; accepted July 7, 2014. This work was supported by the Natural Sciences and Engineering Research Council of Canada, and a Walter C. Sumner Memorial Fellowship.

The authors are with the Department of Electrical and Computer Engineering, University of British Columbia, Vancouver, BC, Canada, V6T $1 \mathrm{Z4}$ (email: \{adamn, kcheung, rschober\}@ece.ubc.ca). R. Schober is also with the Institute for Digital Communication, Friedrich-Alexander-Universität Erlangen-Nürnberg (FAU), Erlangen, Germany (email: schober@lnt.de). of characteristics that distinguish it from conventional communication channels. For example, it features randomly-moving molecules instead of propagating electromagnetic radiation, so the propagation time critically depends on the distance between the transmitter and receiver and there can be significant intersymbol interference (ISI) due to the lingering presence of emitted molecules.

Most existing literature that has studied the potential for transmitting digital information over a diffusive channel has focused on numerically characterizing the capacity in bits per second or bits per use, cf. e.g. [4]-[8]. Recently, an upperbound on capacity was analytically derived in [9]. Various strategies from conventional communications have also been proposed to improve communication via diffusion, including channel coding in [10], emitting Gaussian pulses in [11], and network coding in [12]. However, the feasibility of such strategies is unknown. We anticipate that individual transceivers would have limited computational abilities, therefore it is of interest to consider simple modulation schemes and then assess how simple detectors perform in comparison to optimally designed detectors. Such analysis would provide valuable insight into the practical design of diffusive communication networks.

In this paper, we consider optimal and suboptimal receiver detection schemes for a 3-dimensional stochastic diffusion environment between a single transmitter and receiver. Our physical model includes steady uniform flow in any arbitrary direction, as flow is likely to be present in any environment where molecular communication is deployed; see [1]. We also consider noise sources that release information molecules in addition to those released by the transmitter. Generally, these sources could be interference from similar communication links, or the result of other chemical processes that produce information molecules as their output. We perform a precise characterization of such noise sources in [13]; here, we only assume that their cumulative impact on the receiver can be characterized as a Poisson random variable with time-varying mean (which is sufficient to model either or both of the example cases above). We consider the presence of enzyme molecules in the propagation environment that are capable of degrading the information molecules, which we introduced in [14], [15] as a strategy to mitigate ISI without requiring additional computational complexity at the receiver. The use of enzymes to reduce ISI was also recently proposed in [16].

The primary contributions of this paper are as follows:

1) We derive the mutual information between consecutive observations made by the receiver in order to measure the independence of observations. The assumption of 
independent observations is in general not satisfied, particularly as the time between observations tends to zero. However, we require sample independence for tractability in the design of the optimal detector, so it is essential to develop criteria to measure independence.

2) We derive the optimal sequence detector, in a maximum likelihood sense, to give a lower bound on the bit error probability for any detector at the receiver. In practice, we do not expect such a detector to be physically realizable, due to the memory and computational complexity required, even if the implementation is simplified with the Viterbi algorithm as described in [17].

3) We introduce weighted sum detectors as suboptimal but more physically realizable. In fact, they are used in neurons, which sum inputs from synapses with weights based on the characteristics of each synapse and fire when the sum exceeds a certain threshold; see [18, Ch. 12]. We characterize weighted sum detectors by the number of samples taken by the receiver in each bit interval and the weights assigned to each observation. We derive the expected bit error rate of any weighted sum detector. We show that a particular selection of sample weights is equivalent to a matched filter, and compare this detector with the optimal single bit detector. We also consider equal sample weights as a simpler weight selection scheme that is analogous to energy detection in conventional communication. We note that the receiver in our previous work in [15] can be interpreted as the simplest special case of a weighted sum detector, where an immediate decision is made after only one observation in a given bit interval.

Related work concerning the optimal sequence and single bit detectors was reported in [19], where the authors modeled a 2-dimensional environment without flow or an external noise source. The received signal was analyzed as a Gaussian random variable with time-varying mean, where the transmitter symbols are represented by different molecules. This paper considers modulation with a single type of molecule but with a more general physical model, including the possibility of using enzymes in the propagation environment. We perform the majority of our analysis assuming that the received signal is a Poisson random variable with time-varying mean, which is more easily satisfied and more accurate than the Gaussian approximation. Existing papers that model flow provide detailed analysis but assume that flow is only possible in the direction along a line joining the transmitter and receiver, and furthermore that the physical model itself is one-dimensional, cf. e.g. [7], [20]. This paper does not focus on the impact of flow, but (with the recent exception of [21]) our inclusion of flow in any arbitrary direction has not yet been considered in the literature.

It is important to acknowledge the major simplifying assumptions that are made for our analysis. We do this to highlight the applicability of our work, and also to identify areas for future study. These assumptions are as follows:

1) We assume that the transmitter emits enough molecules for the observations made at the receiver to have a
Poisson distribution 1

2) We assume that the transmitter emits insufficient molecules to change the diffusion coefficient of the propagation medium. Strictly speaking, the diffusion coefficient is a function of the local concentration of information molecules (see [23]), so here the coefficient should vary both spatially and temporally. However, we assume that the information molecules are sufficiently dilute to ignore collisions between them in the propagation environment?

3) The modulation scheme adopted by the transmitter is simple ON/OFF keying with a constant bit interval, where an impulse of molecules is released at the start of an interval to transmit a 1 and the transmitter is "silent" to transmit a 0 . We note that our results can be easily extended to pulse amplitude modulation, where the number of molecules released corresponds to a particular information symbol.

4) We assume an ideal receiver that does not interact with the information molecules but is able to perfectly count the number of those molecules that are within its observation space, as in [9]. We also assume that the receiver is synchronized in time with the transmitter, for example via an external signal as discussed in [24]. We do so in order to focus on the effects of the propagation environment and for tractability. Recent work in [25], [26] has incorporated the impact of a chemical reaction mechanism at the receiver where the observable molecules are the output of a reaction involving the molecules emitted by the transmitter. However, whether such a model can lead to a closed-form time domain expression for the signal observed at the receiver remains an open problem.

5) For the purposes of deriving the mutual information of consecutive receiver observations, we assume that the receiver is spherical (or, equivalently, hemispherical if it is mounted to an infinite plane that forms an elastic boundary of a semi-infinite environment). We focus on the sphere because this shape is naturally formed by membrane bilayers; see [3, Ch. 11]. These bilayers can have molecule receptors embedded in them as part of a functioning nanomachine, such as in a cell.

The rest of this paper is organized as follows. The details of the transmission environment are described in Section [I] In Section III] we present the channel impulse response and describe the time-varying signal that is observed at the receiver. In Section IV, we derive the mutual information between consecutive observations made by the receiver. We derive the optimal sequence detector in a maximum likelihood sense in

\footnotetext{
${ }^{1}$ The number of molecules observed can be most accurately described by the binomial distribution, as we previously discussed in [15], and the Poisson approximation of the binomial distribution is commonly applied when the number of trials is large and the probability of success is small; see [22]. Here, each released molecule represents one trial and the probability of success is that molecule's probability of being observed at the receiver at some time.

${ }^{2}$ In our simulations, the highest information molecule concentration is at the transmitter when the molecules are released. This concentration is $1.67 \mathrm{M}$, which is still much lower than the solvent (water) molecule concentration, which is about $55 \mathrm{M}$.
} 
Section $\mathrm{V}$ assuming independent observations. In Section VI. we introduce weighted sum detectors, which are suboptimal for receiving a sequence of bits, but which may be more easily realizable for bio-inspired nanoscale and microscale communication networks. Numerical results showing detector performance are described in Section VII and conclusions are drawn in Section VIII

\section{SySTEM MOdEL}

There is a transmitter fixed at the origin of an infinite, 3dimensional fluid environment of uniform constant temperature and viscosity. The receiver is a sphere with radius $r_{o b s}$ and volume $V_{o b s}$. It is centered at location $\left\{x_{0}, 0,0\right\}$ (without loss of generality) where $\vec{r}_{0}$ is the vector from the origin to $\left\{x_{0}, 0,0\right\}$. The receiver is a passive observer that does not impede diffusion or initiate chemical reactions. We also note that, by symmetry, the concentrations observed in this environment are equivalent (by a factor of 2) to those in the semiinfinite case where $z \geq 0$ is the fluid environment, the $x y$ plane is an elastic boundary, and the receiver is a hemisphere whose circle face lies on the boundary; see [27, Eq. (2.7)]. This equivalent environment could describe, for example, a small transmitter and receiver mounted along the wall of a blood large vessel. A steady uniform flow (or drift) exists in an arbitrary direction defined by its velocity component along each dimension, i.e., $\left\{v_{x}, v_{y}, v_{z}\right\}$. Analytically, this is the simplest type of flow. Other flows of interest but outside the scope of this work include laminar flow, where successive layers of fluid slide over one another without mixing, as is common in environments such as small blood vessels; see [18 Ch. 5].

The transmitter is a source of information molecules called $A$ molecules. They have a negligible natural degradation rate into $A_{P}$ molecules, but are able to degrade much more quickly if they bind to enzyme molecules called $E$ molecules. We assume that $A$ and $E$ molecules react via the MichaelisMenten reaction mechanism, described as

$$
\begin{aligned}
& E+A \stackrel{k_{1}}{\longrightarrow} E A, \\
& E A \stackrel{k_{-1}}{\longrightarrow} E+A, \\
& E A \stackrel{k_{2}}{\longrightarrow} E+A_{P},
\end{aligned}
$$

where $E A$ is the intermediate formed by the binding of an $A$ molecule to an $E$ molecule, and $k_{1}, k_{-1}$, and $k_{2}$ are the reaction rates for the reactions as shown in (1)-(3) with units molecule ${ }^{-1} \mathrm{~m}^{3} \mathrm{~s}^{-1}, \mathrm{~s}^{-1}$, and $\mathrm{s}^{-1}$, respectively. This mechanism is generally accepted as the fundamental mechanism for enzymatic reactions, though it is not the only mechanism possible; see [28, Ch. 10].

In addition to the transmitter, we assume that the environment has other sources of $A$ molecules, either via interference from other communication links or via some other chemical process that generates $A$ molecules. We only assume that we know the cumulative impact of all noise sources on the received signal, and that we can characterize this impact as a Poisson random variable with time-varying mean. In our simulations, we will limit ourselves to an additive noise variable with constant mean (we perform a more precise characterization of noise and interference in [13]). We emphasize that this additive noise source is distinct from the randomness in the number of $A$ molecules observed by the receiver due to diffusion.

We use a common notation to describe the different molecule types in our model. The number of molecules of species $S$ is given by $N_{S}$, and its concentration at the point defined by vector $\vec{r}$ and at time $t$ in molecule $\cdot \mathrm{m}^{-3}$ is $C_{S}(\vec{r}, t)$ (generally written as $C_{S}$ for compactness). We assume that every molecule diffuses independently of every other molecule, and that every molecule is spherical, so that they all diffuse with constant diffusion coefficient $D_{S}$, found using the Einstein relation as [18, Eq. (4.16)]

$$
D_{S}=\frac{k_{B} T}{6 \pi \eta R_{S}},
$$

where $k_{B}$ is the Boltzmann constant $\left(k_{B}=1.38 \times 10^{-23}\right.$ $\mathrm{J} / \mathrm{K}), T$ is the temperature in kelvin, $\eta$ is the viscosity of the propagation environment, and $R_{S}$ is the molecule radius. We note that the accuracy of the Einstein relation is limited in practice (see [23, Ch. 5]), and we only use it here to get a sense of appropriate values for $D_{S}$; typically, $D_{S}$ is found via experiment. The diffusion (i.e., displacement) of a single molecule along one dimension is a Gaussian random variable with variance $2 D_{S} t$, where $t$ is the diffusing time in seconds; see [18, Eq. (4.6)]. The presence of steady uniform flow means that every diffusing molecule has a net constant displacement due to the flow in addition to the random displacement due to diffusion.

The transmitter has a sequence of binary data $\mathbf{W}=$ $\{W[1], W[2], \ldots\}$ to send to the receiver, where $W[j]$ is the $j$ th information bit and $\operatorname{Pr}(W[j]=1)=P_{1}$. The transmitter uses binary modulation and transmission intervals of duration $T_{\text {int }}$ seconds. To send a binary $1, N_{A_{E M}} A$ molecules are released at the start of the bit interval. To send a binary 0 , no molecules are released. This method is effectively ON/OFF keying, but we place all signal energy at the start of the bit interval in order to mitigate ISI. There is a constant total enzyme concentration $C_{E_{T o t}}$ everywhere. We facilitate simulations by considering $N_{E} E$ molecules that are initially uniformly distributed throughout and bounded within a finite volume $V_{\text {enz }}$ that is sufficiently large to assume that it is infinite in size (although we do not restrict the motion of $A$ molecules to within $V_{e n z}$, and $E A$ molecules probabilistically undergo reaction (2) or (3) if they hit the boundary).

\section{RECEIVER SIGNAL}

The receiver is an ideal passive observer that is synchronized with the transmitter. The signal that it "observes" is a discrete counting signal that represents the number of molecules present within $V_{o b s}$ at the time when the observation is made. This signal, $N_{A o b s}(t)$, has two components: $N_{A T X}(t)$, or the number of observed molecules that originated at the transmitter, and $N_{\text {Anoise }}(t)$, or the number of observed molecules that originated at the additive noise sources. We emphasize that even without additive noise sources, i.e., $\overline{N_{\text {Anoise }}}(t)=0$, $N_{\text {Aobs }}(t)$ will still be noisy due to the random motion of the 
molecules emitted by the transmitter. In this section, we first describe $N_{\text {Aobs }}(t)$ when there is only a single emission by the transmitter in the first bit interval, i.e., the channel impulse response scaled by $N_{A_{E M}}$. Next, we characterize $N_{A o b s}(t)$ as a random variable due to transmitter sequence $\mathbf{W}$. The signal model is a generalization of the receiver signal presented in [15]; here, we add the impacts of flow and external noise sources. We have already assumed that we characterize $N_{\text {Anoise }}(t)$ as a Poisson random variable with time-varying mean, and that the diffusion of molecules is independent. Thus, in the following we only need to characterize $N_{A T X}(t)$.

\section{A. Channel Impulse Response}

The general reaction-diffusion equation for species $S$ is $[29$ Eq. (8.12.1)]

$$
\frac{\partial C_{S}}{\partial t}=D_{S} \nabla^{2} C_{S}+u\left(C_{S}, \vec{r}, t\right),
$$

where $u(\cdot)$ is the reaction term. Using the principles of chemical kinetics as described in [28, Ch. 9], the system of partial differential equations for our environment without flow is [15, Eqs. (6)-(8)]

$$
\begin{aligned}
\frac{\partial C_{A}}{\partial t} & =D_{A} \nabla^{2} C_{A}-k_{1} C_{A} C_{E}+k_{-1} C_{E A} \\
\frac{\partial C_{E}}{\partial t} & =D_{E} \nabla^{2} C_{E}-k_{1} C_{A} C_{E}+k_{-1} C_{E A}+k_{2} C_{E A} \\
\frac{\partial C_{E A}}{\partial t} & =D_{E A} \nabla^{2} C_{E A}+k_{1} C_{A} C_{E}-k_{-1} C_{E A}-k_{2} C_{E A} .
\end{aligned}
$$

To incorporate steady uniform flow, we only need to change the diffusion coefficient terms, as described in [30, Ch. 4]. Specifically, for the $A$ molecules we replace $D_{A} \nabla^{2} C_{A}$ with

$$
D_{A} \nabla^{2} C_{A}-v_{x} \frac{\partial C_{A}}{\partial x}-v_{y} \frac{\partial C_{A}}{\partial y}-v_{z} \frac{\partial C_{A}}{\partial z},
$$

and make analogous substitutions for $E$ and $E A$. The system of equations above has no closed-form analytical solution, even under our boundary conditions of an impulsive point source and unbounded environment. In [15, Eq. (10)], we derived a lower bound on the expected point concentration of $A$ molecules at a distance $|\vec{r}|$ from an impulsive point source that emits at time $t=0$. This lower bound is

$$
C_{A} \geq \frac{N_{A_{E M}}}{\left(4 \pi D_{A} t\right)^{3 / 2}} \exp \left(-k C_{E_{T o t}} t-\frac{|\vec{r}|^{2}}{4 D_{A} t}\right),
$$

where

$$
k=\left\{\begin{aligned}
k_{1} & \text { for a strict lower bound, } \\
\frac{k_{1} k_{2}}{k_{-1}+k_{2}} & \text { for an approximation, }
\end{aligned}\right.
$$

and the approximation for $k$ is much more accurate than the lower bound if $k_{-1}+k_{2} \approx k_{2}$ is not satisfied. The lower bound becomes tight as $k_{2} \rightarrow \infty$ and $k_{-1} \rightarrow 0$, and it is also equivalent to the expected concentration if $A$ molecules degrade directly into $A_{P}$ molecules at rate $k C_{E_{T o t}}$. To include the effect of flow, we simply set $|\vec{r}|^{2}=\left(x-v_{x} t\right)^{2}+\left(y-v_{y} t\right)^{2}+$ $\left(z-v_{z} t\right)^{2}$ for the concentration at point $\{x, y, z\}$.

For clarity of exposition and for tractability in the presence of flow in any direction, we apply the uniform concentration assumption to calculate $N_{A T X}(t)$ from $C_{A}$, where we assume that the expected concentration throughout the receiver is equal to that expected at the center of the receiver. We showed in [31] that the validity of this assumption in the no-flow case improves as the receiver is placed further from the transmitter. We also assume that 10 is satisfied with equality (which is always true when there are no enzymes present) and thus find that the expected channel impulse response at the receiver due to the transmitter, $\overline{N_{A T X}}(t)$, is

$$
\begin{aligned}
\overline{N_{A T X}}(t) & =V_{o b s} C_{A}\left(\sqrt{\left(x_{0}-v_{x} t\right)^{2}+\left(v_{y} t\right)^{2}+\left(v_{z} t\right)^{2}}, t\right) \\
& =V_{o b s} C_{A}\left(\left|\vec{r}_{e f f}\right|, t\right),
\end{aligned}
$$

where $\left|\vec{r}_{e f f}\right|=\sqrt{\left(x_{0}-v_{x} t\right)^{2}+\left(v_{y} t\right)^{2}+\left(v_{z} t\right)^{2}}$ is the effective distance between the transmitter and the center of the receiver, considering the flow.

For a single molecule emitted by the transmitter at time $t=0$, the probability that it is within $V_{o b s}$ at time $t, P_{o b s}(t)$, is given by (12) where $N_{A_{E M}}=1$, i.e.,

$$
P_{o b s}(t)=\frac{V_{o b s}}{\left(4 \pi D_{A} t\right)^{3 / 2}} \exp \left(-k C_{E_{\text {Tot }}} t-\frac{\left|\vec{r}_{e f f}\right|^{2}}{4 D_{A} t}\right) .
$$

We showed in [15] that $N_{A T X}(t)$ due to one emission of $N_{A_{E M}}$ molecules at the transmitter can be accurately approximated as a Poisson random variable with time-varying mean given by $\overline{N_{A T X}}(t)$ in $(12)$. Since $N_{\text {Anoise }}(t)$ is also a Poisson random variable with time-varying mean, then $N_{A o b s}(t)$ is the sum of two Poisson random variables. The sum of independent Poisson random variables is also a Poisson random variable whose mean is the sum of the means of the individual variables; see [22. Ch. 5.2]. Thus, the signal observed is a Poisson random variable with mean $\overline{N_{\text {Anoise }}}(t)+\overline{N_{A T X}}(t)$ with $\overline{N_{A T X}}(t)$ as given by $(12$.

\section{B. Complete Receiver Signal}

In general, the receiver can observe molecules that were emitted at the start of the current or any prior bit interval, depending on $\mathbf{W}$. We assume that the behavior of individual $A$ molecules is independent, so we can immediately write

$$
N_{A T X}(t)=\sum_{j=1}^{\left\lfloor\frac{t}{T_{i n t}}+1\right\rfloor} N_{A T X}(t ; j)
$$

where $N_{A T X}(t ; j)$ is the number of molecules observed at time $t$ that were emitted at the start of the $j$ th bit interval. Of course, $N_{A T X}(t ; j)=0 \forall t$ if $W[j]=0 . N_{A T X}(t)$ is still a sum of Poisson random variables, and so $N_{A o b s}(t)$ is a Poisson random variable with mean

$$
\overline{N_{\text {Aobs }}}(t)=\overline{N_{A T X}}(t)+\overline{N_{\text {Anoise }}}(t),
$$

where

$$
\overline{N_{A T X}}(t)=N_{A_{E M}} \sum_{j=1}^{\left\lfloor\frac{t}{T_{\text {int }}}+1\right\rfloor} W[j] P_{\text {obs }}\left(t-(j-1) T_{\text {int }}\right) .
$$

Thus, the probability density function (PDF) of $N_{A o b s}(t)$ is

$$
\operatorname{Pr}\left(N_{\text {Aobs }}(t)=\xi\right)=\frac{\overline{N_{\text {Aobs }}}(t)^{\xi} \exp \left(-\overline{N_{\text {Aobs }}}(t)\right)}{\xi !},
$$


and the cumulative density function (CDF) is

$$
\operatorname{Pr}\left(N_{\text {Aobs }}(t)<\xi\right)=\exp \left(-\overline{N_{\text {Aobs }}}(t)\right) \sum_{i=0}^{\xi-1} \frac{\overline{N_{A o b s}}(t)^{i}}{i !}
$$

\section{INDEPENDENCE OF RECEIVER OBSERVATIONS}

In this section, we study the assumption that all observations made at the receiver are independent of each other. Intuitively, this will not be true if the time between samples tends to zero; if the time between samples is infinitesimally small, then there is insufficient time for either the molecules observed in one sample to leave the receiver or for any new molecules to arrive. We will measure independence using mutual information, which is a measure of how the knowledge of one variable influences the prediction of a second variable; see [32]. Specifically, for two discrete random variables $X$ and $Y$ taking values $x$ and $y$, respectively, the mutual information $I(X ; Y)$ is [32, Eq. (2.28)]

$$
\begin{aligned}
I(X ; Y)= & \sum_{x} \sum_{y} \operatorname{Pr}(X=x, Y=y) \\
& \times \log \frac{\operatorname{Pr}(X=x, Y=y)}{\operatorname{Pr}(X=x) \operatorname{Pr}(Y=y)},
\end{aligned}
$$

where the summations are over all possible values of $x$ and $y$. If the variables $X$ and $Y$ are independent, then $I(X ; Y)=0$, i.e., knowing $X$ does not provide any greater certainty in predicting the value of $Y$, and vice versa. If $X$ and $Y$ are measures of the same variable over time, and the time between measures is infinitesimally small, then knowing $X$ lets us predict $Y$ perfectly and mutual information is maximized.

We focus on the independence of two consecutive receiver observations, since these are the most likely to be dependent. We define these observations to be at times $t_{1}$ and $t_{2}$, such that $t_{2}>t_{1}$. For clarity, we consider only molecules that were emitted by the transmitter at time $t=0$, so the unconditional time-varying PDF describing whether a molecule is within $V_{o b s}$ is given by $P_{o b s}(t)$ in 13 . Of course, if no molecules are emitted and there are no noise sources, then the mutual information between samples of molecules from the transmitter would be zero because every sample would be 0 . For tractability, we also assume no flow in this section, though we expect that the presence of flow can only decrease the mutual information of the two observations.

Let the observations at times $t_{1}$ and $t_{2}$ be $s_{1}$ and $s_{2}$, respectively. From probability theory, the joint probability distribution of $N_{\text {Aobs }}\left(t_{1}\right)$ and $N_{A o b s}\left(t_{2}\right)$ can be evaluated from the conditional probability distribution, i.e.,

$$
\begin{aligned}
& \operatorname{Pr}\left(N_{\text {Aobs }}\left(t_{1}\right)=s_{1}, N_{\text {Aobs }}\left(t_{2}\right)=s_{2}\right)= \\
& \operatorname{Pr}\left(N_{\text {Aobs }}\left(t_{2}\right)=s_{2} \mid N_{\text {Aobs }}\left(t_{1}\right)=s_{1}\right) \operatorname{Pr}\left(N_{\text {Aobs }}\left(t_{1}\right)=s_{1}\right) .
\end{aligned}
$$

The mutual information is then

$$
\begin{aligned}
& I\left(N_{A_{o b s}}\left(t_{1}\right) ; N_{\text {Aobs }}\left(t_{2}\right)\right)= \\
& \quad \sum_{s_{1}} \sum_{s_{2}} \operatorname{Pr}\left(N_{\text {Aobs }}\left(t_{1}\right)=s_{1}, N_{\text {Aobs }}\left(t_{2}\right)=s_{2}\right) \\
& \quad \times \log \frac{\operatorname{Pr}\left(N_{\text {Aobs }}\left(t_{1}\right)=s_{1}, N_{\text {Aobs }}\left(t_{2}\right)=s_{2}\right)}{\operatorname{Pr}\left(N_{\text {Aobs }}\left(t_{1}\right)=s_{1}\right) \operatorname{Pr}\left(N_{\text {Aobs }}\left(t_{2}\right)=s_{2}\right)},
\end{aligned}
$$

where the range of values for the observations $s_{1}$ and $s_{2}$ in the summations should account for all observations that have a non-negligible probability of occurring. The marginal probabilities (i.e., of a single variable) in (21) can be evaluated using (17) and used to determine appropriate ranges for $s_{1}$ and $s_{2}$. The remaining step to evaluate (21) is to derive the conditional probability in (20). We can derive this probability if we have an expression for $P_{\text {stay }}\left(t_{o}\right)$, the probability that an information molecule that is observed at the receiver at $t_{1}$ is later at the receiver at $t_{o}=t_{2}-t_{1}$. We assume that an information molecule observed at $t_{1}$ is randomly located within the receiver sphere, following the uniform concentration assumption. Then, from [27, Eq. (3.8)], the point concentration $C_{A}\left(r, t_{o}\right)$ due to this single molecule at a distance $r$ from the center of the receiver, without enzymes present, is

$$
\begin{aligned}
C_{A}\left(r, t_{o}\right)= & \frac{3}{8 \pi r_{o b s}^{3}}\left[\operatorname{erf}\left(\frac{r_{o b s}-r}{2 \sqrt{D_{A} t_{o}}}\right)+\operatorname{erf}\left(\frac{r_{o b s}+r}{2 \sqrt{D_{A} t_{o}}}\right)\right] \\
& +\frac{3}{4 \pi r_{o b s}^{3} r} \sqrt{\frac{D_{A} t_{o}}{\pi}}\left[\exp \left(-\frac{\left(r_{o b s}+r\right)^{2}}{4 D_{A} t_{o}}\right)\right. \\
& \left.-\exp \left(-\frac{\left(r_{o b s}-r\right)^{2}}{4 D_{A} t_{o}}\right)\right],
\end{aligned}
$$

where the error function is [33, Eq. (8.250.1)]

$$
\operatorname{erf}(x)=\frac{2}{\pi} \int_{0}^{x} \exp \left(-y^{2}\right) d y,
$$

and we can easily account for enzymes by multiplying 22 by a factor of $\exp \left(-k C_{E_{T o t}} t_{o}\right)$. What follows in the remainder of this section is valid with or without this factor included.

To derive $P_{\text {stay }}\left(t_{o}\right)$, we must integrate $C_{A}\left(r, t_{o}\right)$ over the entire receiver volume, i.e.,

$$
\begin{aligned}
P_{\text {stay }}\left(t_{o}\right) & =\int_{0}^{r_{o b s}} \int_{0}^{2 \pi} \int_{0}^{\pi} C_{A}\left(r, t_{o}\right) r^{2} \sin \theta d \theta d \phi d r \\
& =4 \pi \int_{0}^{r_{o b s}} C_{A}\left(r, t_{o}\right) r^{2} d r .
\end{aligned}
$$

We now present the following theorem:

Theorem $1\left(P_{\text {stay }}\left(t_{o}\right)\right.$ for one molecule): The probability of an information molecule being inside the receiver at time $t_{o}$ after it was last observed inside the receiver is given by

$$
\begin{aligned}
P_{\text {stay }}\left(t_{o}\right)= & \operatorname{erf}\left(\frac{r_{o b s}}{\sqrt{D_{A} t_{o}}}\right)+\frac{1}{r_{o b s}} \sqrt{\frac{D_{A} t_{o}}{\pi}} \\
& \times\left[\left(1-\frac{2 D_{A} t_{o}}{r_{o b s}^{2}}\right) \exp \left(\frac{-r_{o b s}^{2}}{D_{A} t_{o}}\right)+\frac{2 D_{A} t_{o}}{r_{o b s}^{2}}-3\right],
\end{aligned}
$$

where (25) is multiplied by $\exp \left(-k C_{E_{T o t}} t_{o}\right)$ if enzymes are present. 
Proof: Please refer to the Appendix.

The probability that a molecule observed at $t_{1}$ is outside the observer at $t_{2}$ is $P_{\text {leave }}\left(t_{o}\right)=\exp \left(-k C_{E_{\text {Tot }}} t_{o}\right)-P_{\text {stay }}\left(t_{o}\right)$.

To derive an expression for (20), we also require an expression for $P_{a r r}\left(t_{1}, t_{2}\right)$, the unconditional probability that an information molecule that is outside the receiver at $t_{1}$ is inside the receiver at $t_{2}$. Intuitively, this is equal to the unconditional probability of the molecule being inside the receiver at $t_{2}$, minus the probability of the molecule being inside the receiver at $t_{1}$ and still inside the receiver at $t_{2}$, i.e.,

$$
P_{\text {arr }}\left(t_{1}, t_{2}\right)=P_{\text {obs }}\left(t_{2}\right)-P_{\text {obs }}\left(t_{1}\right) P_{\text {stay }}\left(t_{o}\right) .
$$

The conditional probability in (20) must consider every possible number of arrivals and departures of molecules from the receiver in order to result in a net change of $s_{2}-s_{1}$ information molecules. In other words,

$$
\begin{aligned}
& \operatorname{Pr}\left(N_{\text {Aobs }}\left(t_{2}\right)=s_{2} \mid N_{\text {Aobs }}\left(t_{1}\right)=s_{1}\right)= \\
& \sum_{i=\max \left(0, s_{1}-s_{2}\right)}^{s_{1}} \operatorname{Pr}(i \text { leave }) \operatorname{Pr}\left(s_{2}+i-s_{1} \text { arrive }\right),
\end{aligned}
$$

where $\operatorname{Pr}(i$ leave $)$ is conditioned on $N_{\text {Aobs }}\left(t_{1}\right)$ as

$$
\begin{aligned}
& \operatorname{Pr}\left(i \text { leave } \mid N_{\text {Aobs }}\left(t_{1}\right)=s_{1}\right)= \\
& \quad\left(\begin{array}{c}
s_{1} \\
i
\end{array}\right) P_{\text {leave }}\left(t_{o}\right)^{i}\left(1-P_{\text {leave }}\left(t_{o}\right)\right)^{s_{1}-i},
\end{aligned}
$$

and $\operatorname{Pr}(i$ arrive $)$ is also conditioned on $N_{A o b s}\left(t_{1}\right)$ as

$$
\begin{aligned}
& \operatorname{Pr}\left(i \text { arrive } \mid N_{\text {Aobs }}\left(t_{1}\right)=s_{1}\right)= \\
& \quad\left(\begin{array}{c}
N_{A}-s_{1} \\
i
\end{array}\right) P_{\text {arr }}\left(t_{1}, t_{2}\right)^{i}\left[1-P_{\text {arr }}\left(t_{1}, t_{2}\right)\right]^{N_{A}-s_{1}-i} .
\end{aligned}
$$

Eq. (29) can be simplified by applying the Poisson approximation to the binomial distribution and then assuming that $N_{A}-s_{1} \approx N_{A}$, such that 29 ) is no longer conditioned on $s_{1}$ and becomes

$$
\operatorname{Pr}(i \text { arrive })=\frac{\left[N_{A} P_{\text {arr }}\left(t_{1}, t_{2}\right)\right]^{i} \exp \left(-N_{A} P_{\text {arr }}\left(t_{1}, t_{2}\right)\right)}{i !} .
$$

Substituting all components back into 20 enables us to write the two-observation joint probability distribution as

$$
\begin{aligned}
& \operatorname{Pr}\left(N_{\text {Aobs }}\left(t_{1}\right)=s_{1}, N_{\text {Aobs }}\left(t_{2}\right)=s_{2}\right)= \\
& \quad\left[N_{A} P_{\text {obs }}\left(t_{1}\right)\right]^{s_{1}} \exp \left(-N_{A}\left[P_{\text {obs }}\left(t_{1}\right)+P_{\text {arr }}\left(t_{1}, t_{2}\right)\right]\right) \\
& \quad \times \sum_{i=\max \left(0, s_{1}-s_{2}\right)}^{\left.s_{1}\right)} \frac{P_{\text {leave }}\left(t_{o}\right)^{i}\left(1-P_{\text {leave }}\left(t_{o}\right)\right)^{s_{1}-i}}{i !\left(s_{1}-i\right) !} \\
& \times \frac{\left[N_{A} P_{\text {arr }}\left(t_{1}, t_{2}\right)\right]^{s_{2}+i-s_{1}}}{\left(s_{2}+i-s_{1}\right) !} .
\end{aligned}
$$

Using (31) and (17), we can evaluate 211) numerically for any pair of observation times $t_{1}$ and $t_{2}$ and also compare with simulations that generate the joint and marginal probability distributions. We will see in Section VII that as $t_{o}$ increases, $I\left(N_{A_{\text {obs }}}\left(t_{1}\right) ; N_{\text {Aobs }}\left(t_{2}\right)\right)$ decreases, for any value of $t_{1}$.

\section{Optimal Sequence Detection}

In this section, we derive the optimal sequence detector to give a lower bound on the achievable bit error performance of any practical detector. We present a modified version of the Viterbi algorithm to reduce the computational complexity of optimal detection and facilitate its implementation in simulations.

\section{A. Optimal Detector}

The optimal joint interval detection problem can be defined as follows. Let us assume that the transmitter sequence $\mathbf{W}$ is $B$ bits in length. Within each bit interval, the receiver makes $M$ observations. The value of the $m$ th observation in the $j$ th interval is labeled $s_{j, m}$. We assume that the sampling times within a single interval can be written as the function $g(m)$, and we define a global time sampling function $t(j, m)=(j-$ 1) $T_{\text {int }}+g(m)$, where $j=\{1,2, \ldots, B\}, m=\{1,2, \ldots, M\}$. Let us briefly consider two examples of $g(m)$. If a single observation is made when the maximum number of molecules is expected, $t_{\max }$, as we considered in [15], then $g(m)$ has one value, $g(1)=t_{\max }$. If there are observations taken at times separated by constant $t_{o}$, then $g(m)=m t_{o}$.

The optimal receiver decision rule is to select the sequence $\hat{W}[j]$ that is most likely given the joint likelihood of all received samples, i.e.,

$$
\left.\hat{W}[j]\right|_{j=\{1,2, \ldots, B\}}=\underset{W[j], j=\{1,2, \ldots, B\}}{\operatorname{argmax}} \operatorname{Pr}\left(N_{\text {Aobs }}\right)
$$

where

$$
\begin{aligned}
\operatorname{Pr}\left(N_{\text {Aobs }}\right)= & \operatorname{Pr}\left(N_{\text {Aobs }}(t(1,1))=s_{1,1},\right. \\
& N_{\text {Aobs }}(t(1,2))=s_{1,2}, \ldots, \\
& \left.N_{\text {Aobs }}(t(B, M))=s_{B, M} \mid \mathbf{W}\right) .
\end{aligned}
$$

$\operatorname{Pr}\left(N_{A o b s}\right)$ is the joint probability distribution function over all $B M$ observations, given a specified transmitter sequence W. Its form is readily tractable only if we assume that all individual observations are independent of each other, i.e., if

$$
\left.\operatorname{Pr}\left(N_{A_{o b s}}\right)=\prod_{j=1}^{B} \prod_{m=1}^{M} \operatorname{Pr}\left(N_{\text {Aobs }}(t(j, m))=s_{j, m} \mid \mathbf{W}\right)\right) .
$$

If we apply our analysis in Section $[\mathrm{III}$ and conclude that receiver observations are independent, then we can use (34) to determine the likelihood of a given $\mathbf{W}$. However, this is still a problem with significant complexity, especially for large $B$, because we must determine the likelihood of $2^{B}$ possible $\mathbf{W s}$. The total complexity is only linear in the number of samples $M$ since a larger $M$ only means that more terms are included in the product in 34.

\section{B. Optimal Joint Detection Using Viterbi Algorithm}

We consider the Viterbi algorithm in order to reduce the computational complexity of optimal joint detection and evaluate the corresponding probability of error in simulations as a benchmark comparison with simpler detection methods. The memory and computational requirements of the Viterbi 
algorithm are likely still too high for effective implementation in a molecular communication system.

The general Viterbi algorithm is described in detail in [17. Ch. 5]. The algorithm builds a trellis diagram of states where the number of states depends on the channel memory, and each path through the trellis represents one candidate sequence. Our modified implementation artificially shortens the (explicit) channel memory and delays the decision of a given bit by the shortened memory (as performed in methods such as delayed decision-feedback sequence estimation in [34]), but we include the impact of all prior ISI on the current candidate states. If the memory is increased to the actual channel memory, then our method is equivalent to the regular Viterbi algorithm.

Theoretically, the channel memory of a diffusive environment is infinite; from [13, we see that $P_{\text {obs }}(t) \rightarrow 0$ only as $t \rightarrow \infty$. However, in practice, there will be some finite number of bit intervals after which the impact of a given transmission becomes negligible. While it is prudent to include the impact of ISI from all previous bit intervals, we limit the explicit channel memory to $F$ bit intervals. Thus, there will be $2^{F}$ trellis states, where each state represents a candidate sequence for the previous $F$ bit intervals. Each state $f$ has two potential incoming paths, representing the two possible transitions from previous states (each transition corresponds to the possibility of the bit in the $(F+1)$ th prior interval being 0 or 1$)$.

We define $\hat{W}_{f_{i}}[l], l=\{1,2, \ldots, j\}$ as the $l$ th received bit according to the $i$ th path leading to state $f$. The current log likelihood for the $i$ th path leading to state $f$ in the $j$ th interval, which is the likelihood associated with only the observations in the most recent bit interval and the candidate sequence $\hat{W}_{f_{i}}[l]$, is $\Phi_{f_{i}}[j]$ and is evaluated by

$$
\begin{aligned}
\Phi_{f_{i}}[j] & =\sum_{m=1}^{M} \log \left(\operatorname{Pr}\left(N_{\text {Aobs }}(t(j, m))=s_{j, m}\right)\right) \\
& =\sum_{m=1}^{M} \log \left(\frac{\overline{N_{A o b s}}(t)^{s_{j, m}} \exp \left(-\overline{N_{A o b s}}(t)\right)}{s_{j, m} !}\right),
\end{aligned}
$$

where we assume that the observations are independent and we apply the Poisson approximation to the probability of observing a given number of information molecules. We note that taking the logarithm has no influence on the optimality but facilitates numerical evaluation. The cumulative log likelihood for the $f$ th state in the $j$ th interval is the log likelihood of the most likely path (and the corresponding bit sequence $\hat{W}_{f}[l]$ ) to reach the $f$ th state, calculated for all prior bit intervals. We write this likelihood as $L_{f}[j]$ and it is found as

$$
L_{f}[j]=\max \left(L_{f_{1}}[j-1]+\Phi_{f_{1}}[j], L_{f_{2}}[j-1]+\Phi_{f_{2}}[j]\right),
$$

where $L_{f_{i}}[j-1]$ is the cumulative log likelihood of the state prior to the $f$ th state along the $i$ th path. For the $j$ th bit interval, $L_{f}[j]$ is the likelihood associated with the most likely transmitter bit sequence to lead to the $f$ th state. Our modified Viterbi algorithm sequentially builds the trellis diagram by determining $L_{f}[j]$ for every state in every bit interval of the transmission, and keeping track of the candidate bit sequence $\hat{W}_{f}[l]$ that led to $L_{f}[j]$. At the end of the algorithm, the receiver makes its decision by selecting the bit sequence $\hat{W}_{f}[l]$ associated with the largest value of $L_{f}[B]$.

It is straightforward to see that we have reduced the complexity of optimal joint detection by only needing to find the likelihood of $B 2^{F+1}$ sets of $M$ observations, rather than the likelihood of $2^{B}$ sets of $B M$ observations. However, this is still a significant computational burden on the receiver, so it is of interest to consider simpler detection methods. Furthermore, the derivation of the expected bit error probability for a maximum likelihood detector is not easily tractable, so we are restricted to evaluating the bit error probability via simulation.

\section{Weighted Sum Detectors}

In this section, we introduce the family of weighted sum detectors for diffusive molecular communication. These detectors do not have the same memory and computational requirements as maximum likelihood detectors, and we are able to derive the expected bit error probability for a given transmitter sequence.

\section{A. Detector Design and Performance}

We assume that there is only sufficient memory for the receiver to store the $M$ observations made within a single bit interval, and that it is incapable of evaluating likelihoods or considering the impact of prior decisions. Under these limitations, an intuitive receiver design is to add together the individual observations, with a weight assigned to each observation, and then compare the sum with a pre-determined decision threshold. This is the weighted sum detector and it is implemented in neuron-neuron junctions; see [18, Ch. 12]. The detector proposed in our previous work in [15] is the simplest special case of a weighted sum detector, i.e., $M=1$. Under specific conditions, which we will discuss in Section VI-B a particular selection of weights makes the performance of this detector equivalent to the optimal detector described in Section V.

The decision rule of the weighted sum detector in the $j$ th bit interval is

$$
\hat{W}[j]= \begin{cases}1 & \text { if } \sum_{m=1}^{M} w_{m} N_{\text {Aobs }}(t(j, m)) \geq \xi \\ 0 & \text { otherwise, }\end{cases}
$$

where $w_{m}$ is the weight of the $m$ th observation and $\xi$ is the binary decision threshold. For positive integer weights, we only need to consider positive integer decision thresholds.

The method of calculation of the expected error probability is dependent on the selection of the weights. In a special case, if the weights are all equal, particularly if $w_{m}=1 \forall m$, and we assume that the value of each individual observation is a Poisson random variable, then the weighted sum will also be a Poisson random variable whose mean is the sum of the means of the individual observations. Thus, we can immediately write 
the CDF of the weighted sum in the $j$ th bit interval as

$$
\begin{aligned}
& \operatorname{Pr}\left(\sum_{m=1}^{M} N_{\text {Aobs }}(t(j, m))<\xi\right)= \\
& \quad \exp \left(-\sum_{m=1}^{M} \overline{N_{A o b s}}(t(j, m))\right) \\
& \quad \times \sum_{i=0}^{\xi-1} \frac{\left(\sum_{m=1}^{M} \overline{N_{\text {Aobs }}}(t(j, m))\right)^{i}}{i !} .
\end{aligned}
$$

We note that, especially if $M$ is large, relevant values of $\xi$ may be very high, even if the expected number of molecules counted in a single observation is low. Thus, we might have difficulty in evaluating (38) numerically. We present two alternate methods to evaluate (38). First, we can write the CDF of a Poisson random variable $X$ with mean $\lambda$ in terms of Gamma functions, as [35, Eq. (1)]

$$
\operatorname{Pr}(X<x)=\frac{\Gamma(\lceil x\rceil, \lambda)}{\Gamma(\lceil x\rceil)}
$$

for $x>0$, where the Gamma and incomplete Gamma functions are defined by [33, Eq. (8.310.1), Eq. (8.350.2)]

$$
\begin{aligned}
\Gamma(x) & =\int_{0}^{\infty} \exp (-a) a^{x-1} d a, \\
\Gamma(x, \lambda) & =\int_{\lambda}^{\infty} \exp (-a) a^{x-1} d a,
\end{aligned}
$$

respectively. Second, the Gaussian approximation of the Poisson distribution becomes more appropriate as the mean of the Poisson distribution increases. Including a continuity correction (appropriate when approximating a discrete random variable with a continuous random variable; see [22, Ch. 6]), the CDF has the form

$$
\operatorname{Pr}(X<x)=\frac{1}{2}\left[1+\operatorname{erf}\left(\frac{x-0.5-\lambda}{\sqrt{2 \lambda}}\right)\right] .
$$

Now we consider the more general case, where we have positive non-equal weights. Our analysis can then be summarized in the following theorem:

Theorem 2 (Distribution of a weighted sum): Given $\quad M$ Poisson random variables with means $\lambda_{m}$ and non-negative weights $w_{m}$, then the weighted sum $X=\sum_{m=1}^{M} w_{m} X_{m}$ is in general not a Poisson random variable, however the weighted sum of Gaussian approximations of the individual variables is a Gaussian random variable with mean $\sum_{m=1}^{M} w_{m} \lambda_{m}$ and variance $\sum_{m=1}^{M} w_{m}^{2} \lambda_{m}$.

Proof: The proof is straightforward to show using moment generating functions; see [22, Ch. 4]. It can be shown that a sum of weighted independent Poisson random variables is also a Poisson random variable only if the weights $w_{m}$ are all equal to 1 . However, the Gaussian approximation of each $X_{m}$ gives a Gaussian random variable with mean and variance $\lambda_{m}$, and it can be shown that any weighted sum of Gaussian random variables is also a Gaussian random variable.
Using Theorem 2, we can immediately write the CDF of random variable $X=\sum_{m=1}^{M} w_{m} X_{m}$ as

$$
\operatorname{Pr}(X<x)=\frac{1}{2}\left[1+\operatorname{erf}\left(\frac{x-0.5-\sum_{m=1}^{M} w_{m} \lambda_{m}}{\sqrt{2 \sum_{m=1}^{M} w_{m}^{2} \lambda_{m}}}\right)\right] .
$$

In summary, for evaluation of the expected error probability when the weights are equal, we can use the CDF 38, its equivalent form (39), or its approximation (41) where $\lambda=$ $\sum_{m=1}^{M} w_{m} \overline{N_{A \circ b s}}(t(j, m))$. When the weights are not equal, we must use 42 where $\lambda_{m}=\overline{N_{A o b s}}(t(j, m))$.

Given a particular sequence $\mathbf{W}=\{W[1], \ldots, W[B]\}$, we can write the probability of error of the $j$ th bit, $P_{e}[j \mid \mathbf{W}]$, as

$$
\begin{aligned}
& P_{e}[j \mid \mathbf{W}]= \\
& \left\{\begin{array}{l}
\operatorname{Pr}\left(\sum_{m=1}^{M} w_{m} N_{A o b s}(t(j, m))<\xi\right) \\
\operatorname{Pr}\left(\sum_{m=1}^{M} w_{m} N_{A o b s}(t(j, m)) \geq \xi\right)
\end{array} \quad \text { if } W[j]=1,\right.
\end{aligned}
$$

The true expected error probability for the $j$ th bit is found by evaluating (43) for all possible transmitter sequences and scaling each by the likelihood of that sequence occurring in a weighted sum of all error probabilities, i.e.,

$$
\bar{P}_{e}[j]=\sum_{\mathbf{W} \in \mathcal{W}} P_{1}^{n_{1, j}(\mathbf{W})}\left(1-P_{1}\right)^{n_{0, j}(\mathbf{W})} P_{e}[j \mid \mathbf{W}],
$$

where $\mathcal{W}$ is the set of all $2^{B}$ possible sequences and $n_{i, j}(\mathbf{W})$ is the number of occurrences of bit $i$ within the first $j$ bits of the sequence $\mathbf{W}$. In practice, we can randomly generate a large number (e.g., some hundreds or thousands) of sequences based on $P_{1}$, and we will find that it is sufficient to average the probability of error over this subset of total possible sequences.

\section{B. Optimal Weights}

We now consider optimizing the sample weights. It would make sense to assign greater weight to samples that are expected to measure more information molecules. We make this claim more specific in the context of the matched filter.

Consider a signal $h(t)$. The impulse response of the matched filter to signal $h(t)$ is $h\left(T_{\text {int }}-t\right)$, where $T_{\text {int }}$ is the signal interval; see [17, Ch. 5]. The output of such a filter at time $T_{\text {int }}$ is then

$$
\int_{0}^{T_{i n t}} h(\tau) h\left(T_{i n t}-T_{i n t}+\tau\right) d \tau=\int_{0}^{T_{i n t}} h(\tau)^{2} d \tau,
$$

which is the continuous time equivalent of a weighted sum detector where the sample at time $t$ is simply weighted by the expected value of the signal of interest. Thus, we design a matched filter detector by setting the sample weight $w_{m}$ equal to the number of molecules expected from the transmitter, $\overline{N_{A T X}}(g(m))$.

The matched filter is optimal in the sense that it maximizes the signal-to-noise ratio, and it also minimizes the bit error probability if the desired signal is corrupted by additive white Gaussian noise (AWGN). We generally cannot claim that the 
desired signal is corrupted by AWGN, as that would require a large expected number of molecules at all samples and an AWGN external noise source. However, if these conditions were satisfied, and $T_{i n t}$ was chosen to be sufficiently long to ignore the impact of ISI, then the optimal weighted sum detector would have weights $w_{m}=N_{A} P_{o b s}(g(m))$, and it would be equivalent to the optimal sequence detector.

\section{Optimal Decision Threshold}

Our discussion of weighted sum detectors has not included the selection of the decision threshold $\xi$. Obviously, the performance of a particular detector relies on the chosen value of $\xi$. The derivation of the optimal $\xi$ for a weighted sum detector is left for future work. In Section VII when we discuss the bit error probability observed via simulation of a weighted sum detector, we imply that the optimal $\xi$ for the given environment was found via numerical search.

\section{NUMERICAL RESUltS}

In this section, we present simulation results to assess the performance of the detectors described in this paper. Our simulations are executed in a particle-based stochastic framework where time is advanced in discrete steps $\Delta t$. We note that if receiver observations are made at intervals $t_{o}$, then $t_{o}$ must be a multiple of $\Delta t$. For each time step, molecules undergo random motion over continuous space, and the reaction rate constants are used to find the probabilities of reactions (1)(3) occurring within a given step. For further details, please refer to [14], [15]. The inclusion of steady uniform flow as a constant displacement in addition to random diffusion is straightforward, and the impact of additive noise sources is added post-simulation to receiver observations.

In order to focus on a comparison of the performance of the different detectors, most physical parameters remain constant throughout this section. The only environmental parameters that we adjust are the quantity of additive noise, the distance $x_{0}$ to the receiver, the degree of steady uniform flow, and whether enzymes are present. For communication, we vary the length of the bit interval and the frequency of sampling. In the following, we refer to the base case as numerical results found when there is no additive noise, no flow, and no active enzymes present.

We model a fluid environment with a uniform viscosity of $10^{-3} \mathrm{~kg} \cdot \mathrm{m}^{-1} \mathrm{~s}^{-1}$ and at a temperature of $25^{\circ} \mathrm{C}$ (i.e., the solvent is water). The transmitter emits impulses of $N_{A_{E M}}=5000 \mathrm{~A}$ molecules to transmit a binary 1 with probability $P_{1}=0.5$. When enzymes are present, they have a concentration of $84 \mu \mathrm{M}$, and the values of $k_{1}, k_{-1}$, and $k_{2}$ are $2 \times 10^{-19} \frac{\mathrm{m}^{3}}{\text { molecule.s }}, 10^{4} \mathrm{~s}^{-1}$, and $10^{6} \mathrm{~s}^{-1}$, respectively. The radii of the $A, E$, and $E A$ molecules are $0.5 \mathrm{~nm}, 2.5 \mathrm{~nm}$, and $3 \mathrm{~nm}$, respectively (i.e., using (4) we get $D_{A}=4.365 \times 10^{-10} \frac{\mathrm{m}^{2}}{\mathrm{~s}}$ ). Unless otherwise noted, the receiver is centered at $x_{0}=300 \mathrm{~nm}$ and has a radius $r_{o b s}=45 \mathrm{~nm}$. The simulation time step is $\Delta t=0.5 \mu \mathrm{s}$. Although the environment is relatively small (the size of the receiver and its distance from the transmitter are both significantly less than the size of a bacterial cell) with a low number of $A$ molecules and

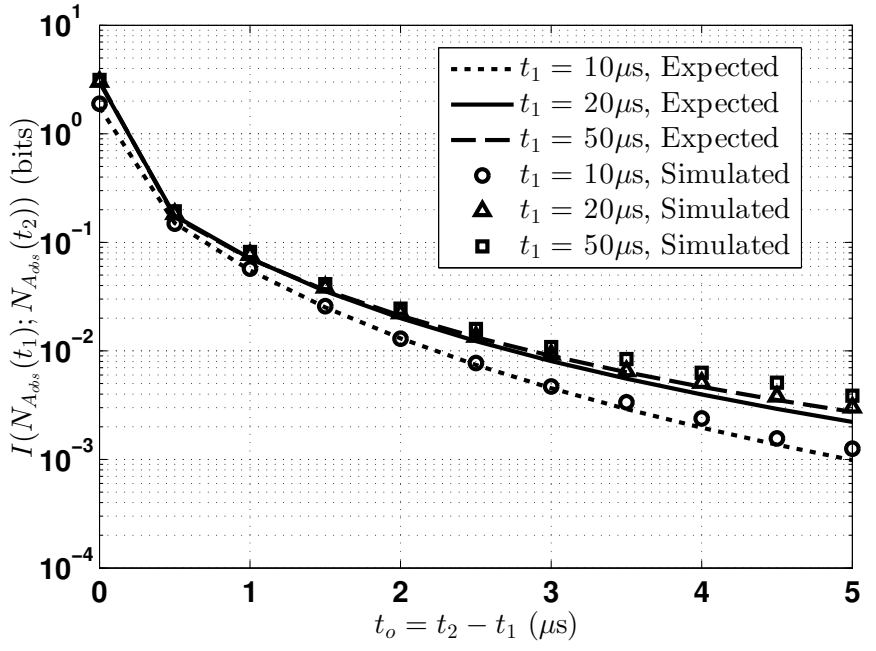

Fig. 1. The mutual information in bits measured as a function of $t_{o}$.

high chemical reactivity, the channel impulse response scales to dimensionally homologous systems that have (for example) more molecules and lower reactivities; see [31]. Our choice of parameters is made to ease the time required for simulations.

\section{A. Sample Independence}

The design of the optimal sequence detector is based on the assumption that all observations made at the receiver are independent of each other. Thus, we consider the mutual information between receiver observations as a function of the time between them, when the only emission of molecules by the transmitter is at time $t=0$. In Fig. 1 . we show the evaluation of the mutual information for the base case when the reference samples are taken at times $t_{1}=\{10,20,50\} \mu \mathrm{s}$. Similar simulation results are observed when flow is present or enzymes are added and we omit these results for clarity. For the expected value curves, we evaluate (21) numerically as described in Section IV] whereas the simulation curves are found by constructing the joint and marginal PDFs using $5 \times 10^{5}$ independent simulations. Base 2 is used in the logarithm so that mutual information is measured in bits.

In Fig. 1, we see that the mutual information drops below 0.01 bits within $4 \mu$ s of all reference samples. The agreement between the expected value curves and those generated via simulations is quite good and only limited by the accuracy of $P_{\text {leave }}(t)$ and $P_{\text {arr }}(t)$ (which critically rely on the uniform concentration assumption).

\section{B. Detector Performance}

We now make a comparison of the average bit error probabilities obtainable via the optimal sequence detector, the matched filter detector, and the equal weight detector. We note that the bit error probability observed for the optimal detector is found assuming independent samples via simulation only. For the matched filter detector, the weights are based on the number of molecules from the transmitter expected at the receiver, $\overline{N_{A T X}}(t)$, due to a transmitter emission in the current interval only (so that the weights are the same 
for every bit interval; the adaptive assignment of weights, which is also performed by neurons as described in [18, Ch. 12], is left for future work). For the equal weight detector, the expected error probability is found using (39). In every subsequent figure, we show the average bit error probability as a function of the number of observations $M$ made per bit interval. For simplicity, observations are equally spaced within the interval, such that $g(m)=m T_{i n t} / M$.

First, we consider the ISI-free case by having the transmitter send a single bit. We do this to assess whether the matched filter performs the same as the optimal detector in the absence of ISI. In order for the detection to be meaningful, we add an independent Poisson noise source with mean 50 and impose a bit interval of $T_{i n t}=200 \mu \mathrm{s}$ for sampling (for reference, the maximum value of $\overline{N_{A T X}}(t)$ under these conditions is 5.20 molecules at $34.36 \mu \mathrm{s}$ after transmission). The bit error probability found via simulation is averaged over $2 \times 10^{5}$ transmissions, and the expected error probability is found considering all bit sequences since in this case there are only two (either 1 or 0 ). The results are presented in Fig. 2 We see that the bit error probability achievable with the matched filter is equal to that of the maximum likelihood detector for any value of $M$. Thus, we claim that the performance of the matched filter is equivalent to the optimal receiver design if there is no ISI, even though the signal is corrupted by non-AWGN noise. Furthermore, the bit error probability achievable with the equal weight detector is greater than those of the optimal detectors. For example, the equal weight detector achieves a bit error probability of 0.03 for $M=100$ samples, whereas the optimal detectors achieve a bit error probability of 0.017 . Finally, the expected error probabilities evaluated for the matched filter and equal weight detectors are very accurate when compared with the simulation results, except when the error probability is below 0.01 (where the deviation is primarily due to the increasing sample dependence). Interestingly, there appears to be no degradation in bit error performance as $t_{o}$ goes to 0 (i.e., as $M$ increases), even for the maximum likelihood detector which was designed assuming independent samples. However, for clarity, and in consideration of Fig. 1. we separate samples in subsequent simulations by at least $5 \mu \mathrm{s}$.

In the subsequent figures, we consider transmitter sequences of 100 consecutive bits, and the error probabilities shown are averaged over all intervals and independent realizations. The expected error probabilities are evaluated by taking the average over 1000 random bit sequences. For optimal joint detection we limit the explicit channel memory to $F=2$ bit intervals as a compromise between computational complexity and observable error probability.

In Fig. 3, we study the impact of adding noise to the base case when the bit interval is $T_{i n t}=200 \mu \mathrm{s}$. Due to ISI, the bit error probability achievable with the matched filter is not as low as that achievable with the optimal sequence detector. The disparity reaches orders of magnitudes as $M$ increases (about 2 orders of magnitude in the noiseless case when $M=20$ ). For all detectors, the error probability rises as additive noise is introduced. However, all detectors are able to achieve a probability of error below 0.01 as more samples are taken, even when $\overline{N_{\text {Anoise }}}(t)=0.5$.

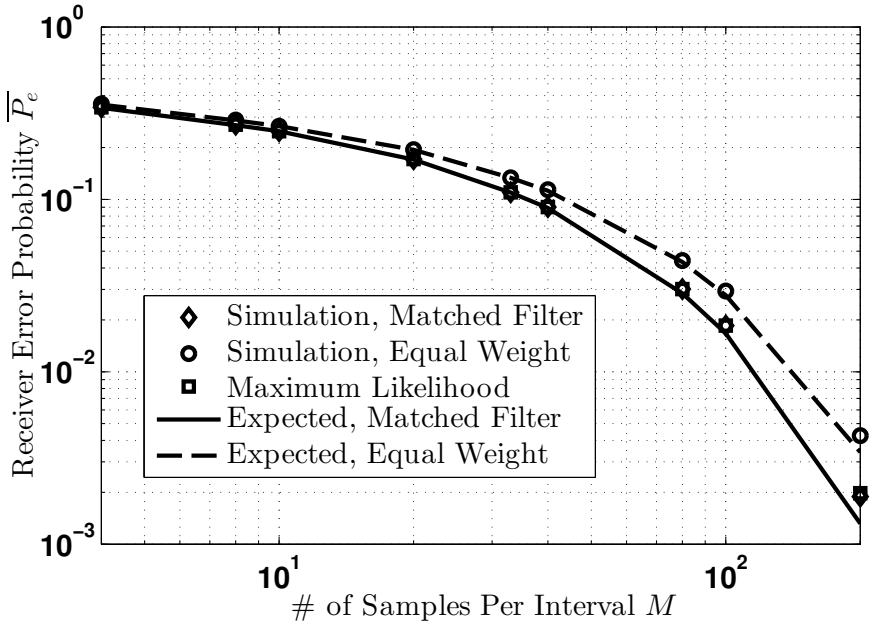

Fig. 2. Expected error probability as a function of $M$ when there is no ISI, $\overline{N_{\text {Anoise }}}(t)=50$, and $T_{\text {int }}=200 \mu \mathrm{s}$. The performance of the matched filter detector is equivalent to that of the maximum likelihood detector.

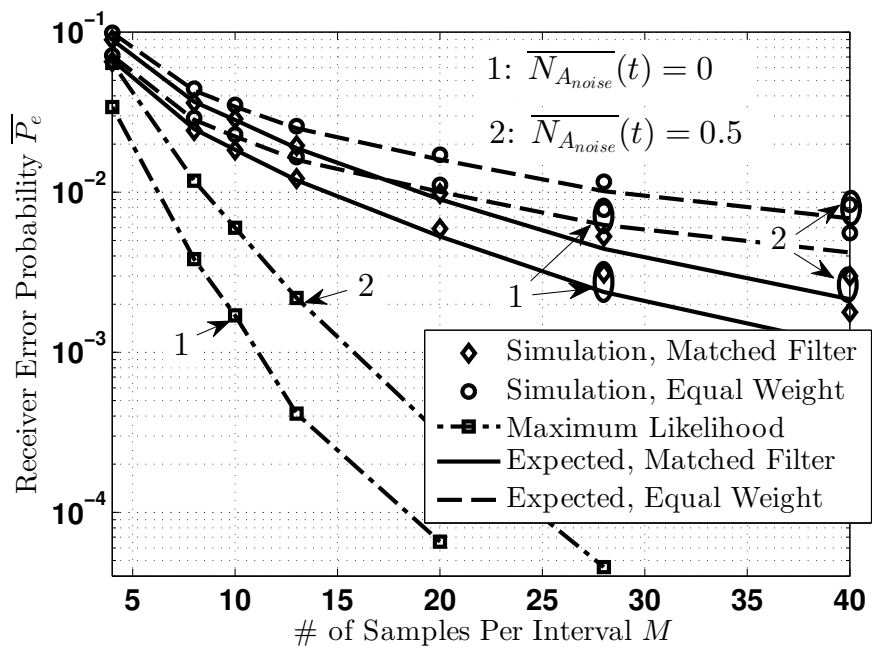

Fig. 3. Receiver error probability as a function of $M$ when ISI is included, $T_{\text {int }}=200 \mu \mathrm{s}$, and $\overline{N_{\text {Anoise }}}(t)=0$ or 0.5 .

In Fig. 4, we consider the impact of propagation distance on receiver performance when the bit interval is $T_{\text {int }}=200 \mu \mathrm{s}$ by varying $x_{0}$ from $250 \mathrm{~nm}$ to $500 \mathrm{~nm}$ while keeping all other transmission parameters constant. Noise is not added. We see that all detectors are very sensitive to the propagation distance; the bit error probability varies over many orders of magnitude, even though the distances vary at most by a factor of 2 . As the distance increases, fewer molecules reach the receiver (i.e., there is less received signal energy) and it takes more time for those molecules to arrive (i.e., the channel impulse response is longer and there is relatively more ISI, although the optimal sequence detector is more robust to this effect). The performance degradation at longer distances can be mitigated by methods such as increasing the bit interval time, changing the number of molecules released by the transmitter, or adding enzymes to the propagation environment.

In Figs. 5 and 6 , we consider the impact of the presence of enzymes in the propagation environment in comparison with 


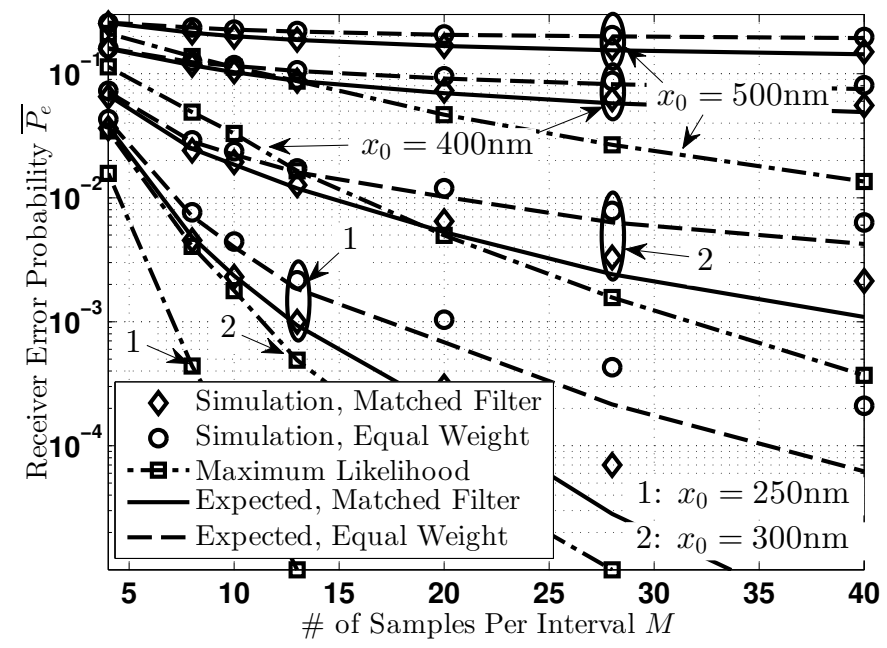

Fig. 4. Receiver error probability as a function of $M$ when ISI is included, $T_{\text {int }}=200 \mu \mathrm{s}$, and the distance $x_{0}$ to the receiver is varied.

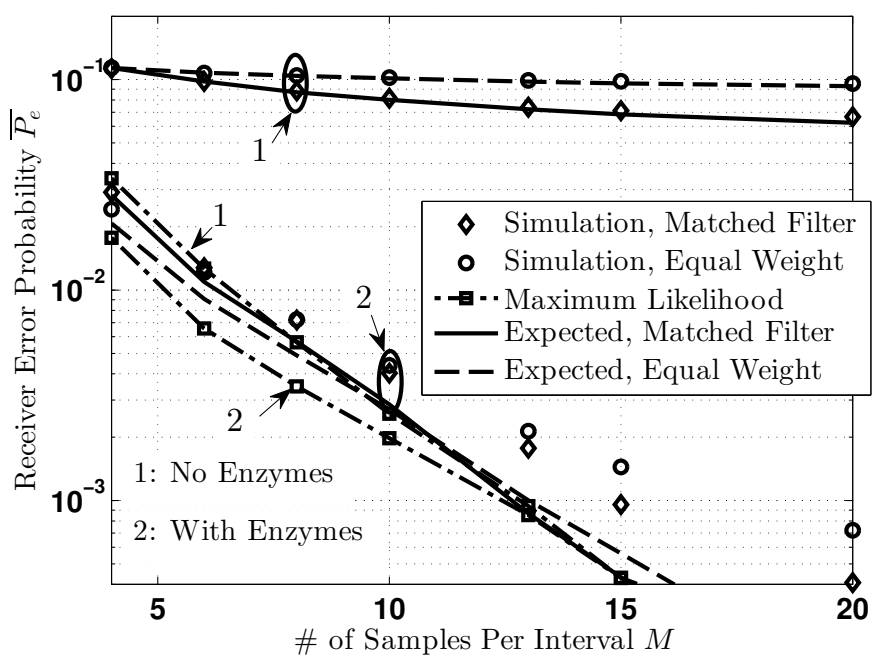

Fig. 5. Receiver error probability as a function of $M$ when ISI is included, $T_{\text {int }}=100 \mu \mathrm{s}$, and enzymes are added to mitigate the impact of ISI.

the base case when the bit interval is $T_{\text {int }}=100 \mu \mathrm{s}$, i.e., shorter than that in Figs. 3 and 4 . In both Figs. 5 and 6 , the expected error probabilities when enzymes are present are not as accurate as in the base case. This is because we assumed that the concentration expected at the receiver as given in (10) is exact, when in fact it is a lower bound (we used $k_{1}$ for $k$ ). As the expected error probability decreases, it becomes relatively more sensitive to the accuracy of this bound.

In Fig. 5, we consider no additive noise sources. The bit interval is so short that, without enzymes, the bit error probability observed by the weighted sum detectors reaches a floor of about 0.06. Orders of magnitude of improvement in bit error probability are observed when enzymes are added to degrade the $A$ molecules and mitigate the impact of ISI; both weighted sum detectors give a bit error probability below 0.005 for $M \geq 10$, and performance is within an order of magnitude of the maximum likelihood detector. Interestingly, the equal weight detector outperforms the matched filter

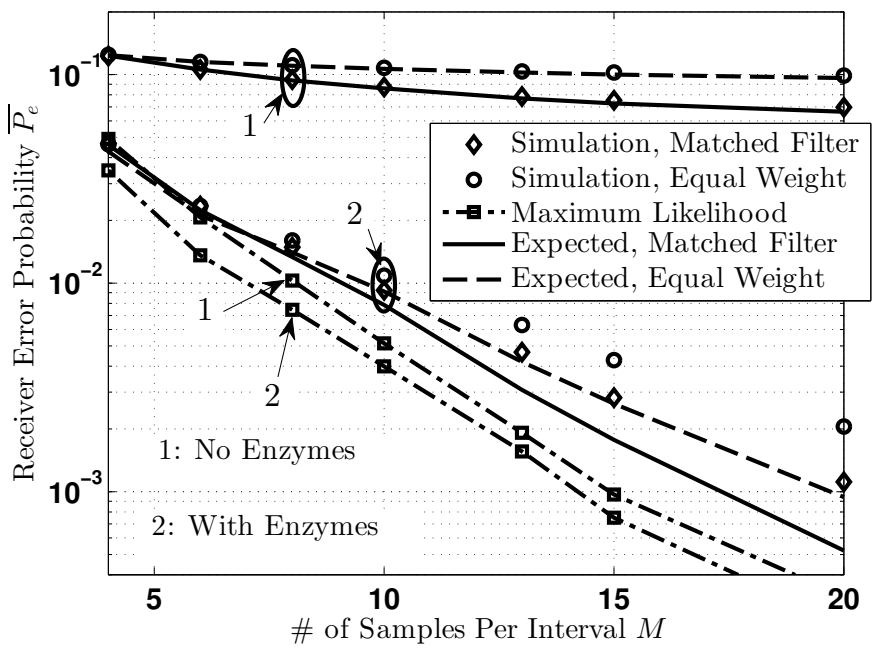

Fig. 6. Receiver error probability as a function of $M$ when ISI is included, $T_{i n t}=100 \mu \mathrm{s}$, enzymes are added to mitigate the impact of ISI, and an additive noise source is present $\overline{\left(N_{\text {Anoise }}\right.}(t)=1$ without enzymes and $\overline{N_{\text {Anoise }}}(t)=0.5$ when enzymes are present).

detector for $M<10$, emphasizing that the matched filter does not necessarily optimize the probability of error when the noise is not AWGN and ISI is present (though the Gaussian approximation of the noise improves as $M$ increases). There is also an improvement in the performance of the maximum likelihood detector for the range of $M$ shown, even though the enzymes are effectively destroying signal energy that could have been used to help with joint detection (the maximum likelihood detector performs better without enzymes when $M>15$, but this is omitted from Fig. 5 to maintain clarity). The reason for this improvement is the chosen value of $F=2$; the actual channel memory is much longer without enzymes.

In Fig. 6, we include an additive noise source. Since we do not model the actual location of the noise source, we cannot predict how much of this noise will be broken down by enzymes before it reaches the receiver. Intuitively, the enzymes will be able to more easily degrade the molecules emitted by the noise source before they are observed by the receiver if the noise source is placed further away. For a fair comparison, we consider a noise source that is placed at a "moderate" distance from the receiver, such that the receiver observes noise with mean 1 in the base case and with mean 0.5 when enzymes are present. The optimal detector can now clearly benefit from the presence of enzymes for all values of $M$, since the enzymes break down noise molecules in addition to those emitted by the transmitter. The improvement in bit error probability of the optimal detector is about $20 \%$ when enzymes are present, for all values of $M$. Of course, the error probabilities observed by all detectors either with or without enzymes are worse than those observed in the no-noise case in Fig. 5

In Fig. 7, we consider the impact of flow when there is an additive noise source with mean value 1 (we assume that the amount of additive noise observed is independent of the flow, but of course in practice that would depend on the nature of the noise source). We set the bit interval to $T_{\text {int }}=100 \mu \mathrm{s}$, the same as in Fig. 6, so we can make 


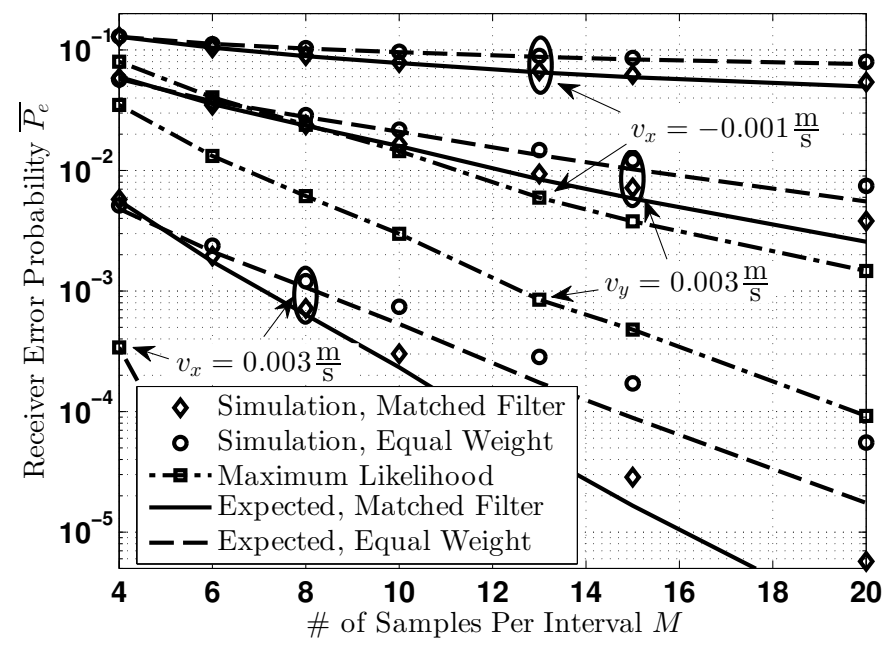

Fig. 7. Receiver error probability as a function of $M$ when ISI is included, $T_{\text {int }}=100 \mu \mathrm{s}, \overline{N_{\text {Anoise }}}(t)=1$, and different degrees of flow are present.

comparisons with the detectors in Fig. 6. We plot the average error probability for three different flows: $v_{x}=0.003 \mathrm{~m} / \mathrm{s}$ (i.e., towards the receiver from the transmitter), $v_{x}=-0.001 \mathrm{~m} / \mathrm{s}$, and $v_{y}=0.003 \mathrm{~m} / \mathrm{s}$ (i.e., perpendicular to the line between transmitter and receiver). The flow magnitudes are chosen so that they affect but do not dominate the channel impulse response (the corresponding Peclet numbers, which describe the dominance of convection versus diffusion and are found here as $\frac{x_{0} v}{D_{A}}$, are 2.06 for $v_{y}$ and the positive $v_{x}$; see [18. Ch. 5]). Of course, for a given flow, the random diffusion of each molecule in every time step is added to the constant displacement due to flow.

When $v_{x}$ is positive, the observed error probabilities in Fig. 7 are much better than in the corresponding no-enzyme case in Fig. 6, and also better than when enzymes are present, as the flow both increases the strength of the observed signal and mitigates ISI. The expected performance of the weighted sum detectors is not as accurate for positive $v_{x}$ because the uniform concentration assumption at the receiver has even less validity with this flow. Perhaps more interestingly, we see that the observed error probabilities with the weighted sum detectors are better than the no-enzyme case in Fig. 6 when $v_{x}$ is negative (although the magnitude of negative flow that we consider is less than that of the positive flow, such that we are still able to observe $A$ molecules at the receiver). Furthermore, all detectors perform better than the no-enzyme case when the direction of flow is perpendicular to the direction of information transmission. These "disruptive" flows, which are not in the direction of information transmission, do not prevent the ability to communicate, and in these cases improve transmission. Thus, it is possible to consider bidirectional transmission in flowing environments, independent of the direction of the flow. We conduct further study on the impact of steady uniform flows with a broader range of Peclet numbers in [36].

\section{CONCLUSION}

In this paper, we studied both optimal and suboptimal detectors for an ideal receiver in a diffusive molecular communication environment. Our physical model is a general one that can include steady uniform flow in any arbitrary direction, sources of information molecules in addition to the transmitter, and enzymes in the propagation environment to degrade the information molecules. We derived the mutual information between receiver observations to show how often independent observations can be made. Furthermore, we derived the maximum likelihood sequence detector to provide a lower bound on the achievable bit error probability. We also designed weighted sum detectors as a family of more practical detectors, where the optimal selection of weights under corruption by AWGN is the matched filter and it performs very close to the optimal detector in the absence of ISI, even if the additive noise is not Gaussian. Simpler weighted sum detectors, with either equal weights or fewer samples per bit interval, offer an easier implementation at the cost of higher error probability. We showed that having enzymes present enables high throughput without relying on the complexity of the optimal detector. We also showed that communication using weighted sum detectors can be improved in flowing environments, for any steady flow direction, so long as the velocity of the flow is not too high.

\section{APPENDIX}

The integration in 24) to prove Theorem 1 can be divided into three parts, which we write as

$$
\begin{aligned}
& \frac{3}{2 r_{o b s}^{3}} \int_{0}^{r_{o b s}} r^{2}\left(\operatorname{erf}\left(\frac{r_{o b s}-r}{2 \sqrt{D_{A} t_{o}}}\right)+\operatorname{erf}\left(\frac{r_{o b s}+r}{2 \sqrt{D_{A} t_{o}}}\right)\right) d r \\
& -\frac{3}{r_{o b s}^{3}} \sqrt{\frac{D_{A} t_{o}}{\pi}} \int_{0}^{r_{o b s}} r \exp \left(-\frac{\left(r_{o b s}-r\right)^{2}}{4 D_{A} t_{o}}\right) d r \\
& \frac{3}{r_{o b s}^{3}} \sqrt{\frac{D_{A} t_{o}}{\pi}} \int_{0}^{r_{o b s}} r \exp \left(-\frac{\left(r_{o b s}+r\right)^{2}}{4 D_{A} t_{o}}\right) d r
\end{aligned}
$$

We begin with (47). It is straightforward to show via the substitution $x=r_{o b s}-r$ and the definition of the error function in (23) that 47) evaluates to

$$
-\frac{3 D_{A} t_{o}}{r_{o b s}^{2}} \operatorname{erf}\left(\frac{r_{o b s}}{2 \sqrt{D_{A} t_{o}}}\right)-\frac{6\left(D_{A} t_{o}\right)^{\frac{3}{2}}}{r_{o b s}^{3} \sqrt{\pi}}\left[\exp \left(\frac{-r_{o b s}^{2}}{4 D_{A} t_{o}}\right)-1\right] .
$$

Similarly, 48 evaluates to

$$
\begin{aligned}
& \frac{6\left(D_{A} t_{o}\right)^{\frac{3}{2}}}{r_{o b s}^{3} \sqrt{\pi}}\left[\exp \left(\frac{-r_{o b s}^{2}}{4 D_{A} t_{o}}\right)-\exp \left(\frac{-r_{o b s}^{2}}{D_{A} t_{o}}\right)\right] \\
& \quad+\frac{3 D_{A} t_{o}}{r_{o b s}^{2}}\left[\operatorname{erf}\left(\frac{r_{o b s}}{2 \sqrt{D_{A} t_{o}}}\right)-\operatorname{erf}\left(\frac{r_{o b s}}{\sqrt{D_{A} t_{o}}}\right)\right] .
\end{aligned}
$$

To solve (46), we first apply the substitutions $y=\left(r_{o b s} \pm\right.$ $r) /\left(D_{A} t_{o}\right)$ to integrals containing the first and second error 
functions, respectively. This enables us to rewrite w6 with a single error function, as

$$
\frac{3}{2 r_{o b s}^{3}} \int_{0}^{\frac{r_{o b s}}{\sqrt{D_{A} t_{o}}}}\left(r_{o b s}-2 y \sqrt{D_{A} t_{o}}\right)^{2} \operatorname{erf}(y) d y .
$$

Evaluating 51) requires the solution of three integrals, being the product of $\operatorname{erf}(y)$ with increasing powers of $y$. Beginning with the base case, from [33, Eq. (5.41)] we have

$$
\int \operatorname{erf}(y) d y=y \operatorname{erf}(y)+\frac{1}{\sqrt{\pi}} \exp \left(-y^{2}\right) .
$$

All terms in (51) can be evaluated using (52) and integration by parts, such that 46 evaluates to

$$
\begin{aligned}
\frac{1}{r_{o b s}} \sqrt{\frac{D_{A} t_{o}}{\pi}}[(1+ & \left.\left.\frac{4 D_{A} t_{o}}{r_{o b s}^{2}}\right) \exp \left(\frac{-r_{o b s}^{2}}{D_{A} t_{o}}\right)-3+\frac{4 D_{A} t_{o}}{r_{o b s}^{2}}\right] \\
& +\left[\frac{3 D_{A} t_{o}}{r_{o b s}^{2}}+1\right] \operatorname{erf}\left(\frac{r_{o b s}}{\sqrt{D_{A} t_{o}}}\right) \cdot
\end{aligned}
$$

It is straightforward to combine (49, ,50, and 53, to arrive at 25.

\section{REFERENCES}

[1] I. F. Akyildiz, F. Brunetti, and C. Blazquez, "Nanonetworks: A new communication paradigm," Computer Networks, vol. 52, no. 12, pp. 2260-2279, May 2008.

[2] T. Nakano, M. J. Moore, F. Wei, A. V. Vasilakos, and J. Shuai, "Molecular communication and networking: Opportunities and challenges," IEEE Trans. Nanobiosci., vol. 11, no. 2, pp. 135-148, Jun. 2012.

[3] B. Alberts, D. Bray, K. Hopkin, A. Johnson, J. Lewis, M. Raff, K. Roberts, and P. Walter, Essential Cell Biology, 3rd ed. Garland Science, 2010.

[4] B. Atakan and O. B. Akan, "Deterministic capacity of information flow in molecular nanonetworks," Nano Commun. Net., vol. 1, no. 1, pp. 31-42, Mar. 2010.

[5] M. S. Kuran, H. B. Yilmaz, T. Tugcu, and I. F. Akyildiz, "Modulation techniques for communication via diffusion in nanonetworks," in Proc. IEEE ICC, Jun. 2011, pp. 1-5.

[6] A. Einolghozati, M. Sardari, and F. Fekri, "Capacity of diffusion-based molecular communication with ligand receptors," in Proc. IEEE ITW, Oct. 2011, pp. 85-89.

[7] S. Kadloor, R. R. Adve, and A. W. Eckford, "Molecular communication using Brownian motion with drift," IEEE Trans. Nanobiosci., vol. 11, no. 2, pp. 89-99, Jun. 2012.

[8] A. W. Eckford, K. V. Srinivas, and R. S. Adve, "The peak constrained additive inverse Gaussian noise channel," in Proc. IEEE ISIT, Jul. 2012, pp. 2973-2977.

[9] M. Pierobon and I. F. Akyildiz, "Capacity of a diffusion-based molecular communication system with channel memory and molecular noise," IEEE Trans. Inf. Theory, vol. 59, no. 2, pp. 942-954, Feb. 2013.

[10] P.-J. Shih, C.-H. Lee, and P.-C. Yeh, "Channel codes for mitigating intersymbol interference in diffusion-based molecular communications," in Proc. IEEE GLOBECOM, Dec. 2012, pp. 4444-4448.

[11] M. Pierobon and I. F. Akyildiz, "Intersymbol and co-channel interference in diffusion-based molecular communication," in Proc. IEEE ICC MONACOM, Jun. 2012, pp. 6126-6131.

[12] B. D. Unluturk, D. Malak, and O. B. Akan, "Rate-delay tradeoff with network coding in molecular nanonetworks," IEEE Trans. Nanotechnol., vol. 12, no. 2, pp. 120-128, Mar. 2013.

[13] A. Noel, K. C. Cheung, and R. Schober, "A unifying model for external noise sources and ISI in diffusive molecular communication," To appear in IEEE J. Sel. Areas Commun., 2014. [Online]. Available: arXiv: 1310.5930

[14] -, "Improving diffusion-based molecular communication with unanchored enzymes," in Proc. ICST BIONETICS, Dec. 2012, pp. 1-15. [Online]. Available: arXiv:1305.1783
[15] _ - "Improving receiver performance of diffusive molecular communication with enzymes," IEEE Trans. Nanobiosci., vol. 13, no. 1, pp. 31-43, Mar. 2014.

[16] M. S. Kuran, H. B. Yilmaz, and T. Tugcu, "A tunnel-based approach for signal shaping in molecular communication," in Proc. IEEE ICC MONACOM, Jun. 2013, pp. 776-781.

[17] J. G. Proakis, Digital Communications, 4th ed. Boston: McGraw-Hill, 2001.

[18] P. Nelson, Biological Physics: Energy, Information, Life, updated 1st ed. W. H. Freeman and Company, 2008.

[19] H. ShahMohammadian, G. G. Messier, and S. Magierowski, "Optimum receiver for molecule shift keying modulation in diffusion-based molecular communication channels," Nano Commun. Net., vol. 3, no. 3, pp. 183-195, Sep. 2012.

[20] K. V. Srinivas, A. W. Eckford, and R. S. Adve, "Molecular communication in fluid media: The additive inverse Gaussian noise channel," IEEE Trans. Inf. Theory, vol. 58, no. 7, pp. 4678-4692, Jul. 2012.

[21] H. ShahMohammadian, G. G. Messier, and S. Magierowski, "Nanomachine molecular communication over a moving propagation medium," Nano Commun. Net., vol. 4, no. 3, pp. 142-153, Sep. 2013.

[22] S. Ross, Introduction to Probability and Statistics for Engineers and Scientists, 4th ed. Academic Press, 2009.

[23] E. L. Cussler, Diffusion: Mass transfer in fluid systems. Cambridge University Press, 1984.

[24] M. J. Moore, T. Nakano, A. Enomoto, and T. Suda, "Measuring distance from single spike feedback signals in molecular communication," IEEE Trans. Signal Process., vol. 60, no. 7, pp. 3576-3587, Jul. 2012.

[25] H. ShahMohammadian, G. G. Messier, and S. Magierowski, "Modelling the reception process in diffusion-based molecular communication channels," in Proc. IEEE ICC MONACOM, Jun. 2013, pp. 782-786.

[26] C. T. Chou, "Extended master equation models for molecular communication networks," IEEE Trans. Nanobiosci, vol. 12, no. 2, pp. 79-92, Jun. 2013.

[27] J. Crank, The Mathematics of Diffusion, 2nd ed. Oxford University Press, 1980.

[28] R. Chang, Physical Chemistry for the Biosciences. University Science Books, 2005.

[29] L. Debnath, Nonlinear Partial Differential Equations for Scientists and Engineers, 2nd ed. Birkhaeuser, 2005.

[30] H. C. Berg, Random Walks in Biology. Princeton University Press, 1993.

[31] A. Noel, K. C. Cheung, and R. Schober, "Using dimensional analysis to assess scalability and accuracy in molecular communication," in Proc. IEEE ICC MONACOM, Jun. 2013, pp. 818-823.

[32] T. M. Cover and J. A. Thomas, Elements of Information Theory, 2nd ed. Wiley-Interscience, 2006.

[33] I. S. Gradshteyn and I. M. Ryzhik, Table of Integrals, Series, and Products, 5th ed. London: Academic Press, 1994.

[34] A. Duel-Hallen and C. Heegard, "Delayed decision-feedback sequence estimation," IEEE Trans. Commun., vol. 37, no. 5, pp. 428-436, May 1989.

[35] A. Ilienko, "Continuous counterparts of poisson and binomial distributions and their properties," Annales Univ. Sci. Budapest, Sect. Comp., vol. 39, pp. 137-147, 2013.

[36] A. Noel, K. C. Cheung, and R. Schober, "Diffusive molecular communication with disruptive flows," in Proc. IEEE ICC, Jun. 2014, pp. 1-7. [Online]. Available: arXiv:1309.5201

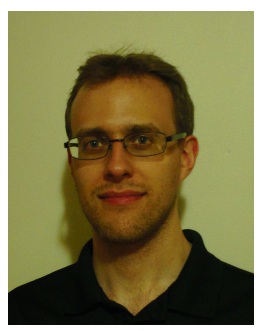

Adam Noel (S'09) received the B.Eng. degree from Memorial University in 2009 and the M.A.Sc. degree from the University of British Columbia (UBC) in 2011, both in electrical engineering. He is now a $\mathrm{Ph} . \mathrm{D}$. candidate in electrical engineering at UBC, and in 2013 was a visiting researcher at the Institute for Digital Communications, Friedrich-AlexanderUniversität Erlangen-Nürnberg. His research interests include wireless communications and how traditional communication theory applies to molecular communication. 


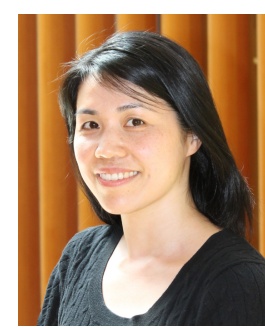

Karen C. Cheung received the B.S. and Ph.D. degrees in bioengineering from the University of California, Berkeley, in 1998 and 2002, respectively. From 2002 to 2005, she was a postdoctoral researcher at the Ecole Polytechnique Fdrale de Lausanne, Lausanne, Switzerland. She is now at the University of British Columbia, Vancouver, BC, Canada. Her research interests include lab-on-a-chip systems for cell culture and characterization, inkjet printing for tissue engineering, and implantable neural interfaces.

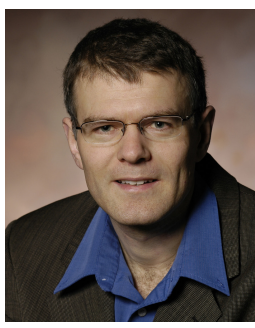

Robert Schober (S'98, M'01, SM'08, F'10) received the Diplom (Univ.) and the Ph.D. degrees in electrical engineering from the University of Erlangen-Nuremberg in 1997 and 2000, respectively. Since May 2002 he has been with the University of British Columbia (UBC), Vancouver, Canada, where he is now a Full Professor. Since January 2012 he is an Alexander von Humboldt Professor and the Chair for Digital Communication at the Friedrich Alexander University (FAU), Erlangen, Germany. His research interests fall into the broad areas of Communication Theory, Wireless Communications, and Statistical Signal Processing. He is currently the Editor-in-Chief of the IEEE Transactions on Communications. 